\title{
Fe emission and ionized excess absorption in the luminous quasar 3C 109 with XMM-Newton
}

\author{
G. Miniutti ${ }^{1 \star}$, D. R. Ballantyne ${ }^{2}$, S. W. Allen ${ }^{3}$, A. C. Fabian ${ }^{1}$ and R. R. Ross ${ }^{4}$ \\ ${ }^{1}$ Institute of Astronomy, Madingley Road, Cambridge CB3 OHA \\ ${ }^{2}$ Department of Physics, University of Arizona, 1118 East 4th Street, Tucson, AZ 85721 USA \\ ${ }^{3}$ Kavli Institute for Particle Astrophysics and Cosmology, Stanford University, 382 Via Pueblo Mall, Stanford, CA 94305-4060, USA \\ ${ }^{4}$ Physics Department, College of the Holy Cross, Worcester, MA 01610, USA
}

31 May 2006

\begin{abstract}
We report results from an XMM-Newton observation of the broad-line radio galaxy 3C 109 $(\mathrm{z}=0.3056$ ). Previous $A S C A$ data revealed the presence of a broad iron line from the accretion disc with which the $X M M-N e w t o n$ spectrum is fully consistent. However, although improving the ASCA constraints on the line parameters, the quality of the data is not high enough to distinguish between an untruncated accretion disc extending down to small radii close to the black hole and a scenario in which the innermost 20-30 gravitational radii are missing. For this reason, our results are model-dependent and the hard data can be modelled equally well by considering an absorption scenario in which a large column of neutral gas partially covers the $\mathrm{X}$-ray continuum source. However, the absorber would have to comprise hundreds/thousands very compact clouds close to the $\mathrm{X}$-ray source, which seems rather extreme a requirement. The $2-10 \mathrm{keV}$ intrinsic luminosity of $3 \mathrm{C} 109$ is of the order of $2-3 \times 10^{45} \mathrm{erg} \mathrm{s}^{-1}$ regardless of the adopted model. A recent black hole mass estimate of $\sim 2 \times 10^{8} M_{\odot}$ implies that $L_{\mathrm{bol}} / L_{\mathrm{Edd}}>1$. If partial covering is excluded, the observed reflection fraction (of the order of unity), steep photon index (1.86), and Fe line equivalent width (about $100 \mathrm{eV}$ ) all suggest to exclude that the $\mathrm{X}$-ray continuum is strongly beamed indicating that the large Eddington ratio is associated with a radiatively efficient accretion process and making it unlikely that the innermost accretion disc is replaced by a thick radiatively inefficient medium such as in advection-dominated accretion models. We also confirm previous findings on the detection of low energy absorption in excess of the Galactic value, where we find excellent agreement with previous results obtained in X-rays and at other wavelengths (optical and infrared). The better quality of the $X M M-$ Newton data enables us to attribute the excess absorption to slightly ionized gas in the line of sight, located at the redshift of 3C 109. The most likely interpretation for the excess absorption is that the line-of-sight is grazing the obscuring torus of unified models, which is consistent with the inclination inferred from the Fe line profile (about $40^{\circ}$ ) and with the hybrid radio-galaxy/quasar nature of 3C 109.
\end{abstract}

Key words: galaxies: active - quasars: individual: 3C 109 - X-rays: galaxies - X-rays: individual: 3C 109

\section{INTRODUCTION}

According to orientation-dependent unified models, radio-loud quasars and radio galaxies belong to the same parent population and their observed properties depend on our viewing angle with respect to the obscuring torus and jet axis (e.g. Orr \& Browne 1982; Barthel 1989; Antonucci 1993). The dusty torus hides the active nucleus and broad-line region in radio galaxies, whereas these are viewed directly in quasars. The half opening angle of the torus is thus the critical angle (about $45^{\circ}$, e.g. Barthel 1989) at which the

^ miniutti@ast.cam.ac.uk transition between radio galaxies and quasars is thought to occur. However, given the observed increase of quasar fraction with distance and luminosity (Singal 1996; Willot et al. 2000), the opening angle is likely to increase with luminosity (e.g. the receding torus model, Lawrence 1991; Simpson 2003 for a review) allowing the broad-line region to be seen at the highest luminosities (but see e.g. Haas et al. 2004).

3C 109 is an example of a luminous active galactic nucleus (AGN) with observed properties placing it at the boundaries between broad-line radio galaxies (BLRGs) and quasars. In the optical 3C 109 appears as a faint galaxy at $\mathrm{z}=0.3056$ (Spinrad et al. 1985 ) with an unresolved nucleus. Early optical studies revealed a 
large Balmer decrement and a red continuum (Yee \& Oke 1978; Grandi \& Osterbrock 1978). Rudy et al. (1984) found strong and non-variable optical polarisation (about 8 per cent) in both the optical continuum and $\mathrm{H} \alpha$ emission. Such a level of polarisation, if due to transmission through aligned dust grains, is consistent with a large intrinsic reddening of the order of $E(B-V)=1.0-1.5$, a result later consolidated through spectro-polarimetric observations by Goodrich \& Cohen (1992) who derived a total continuum reddening of $\mathrm{E}(\mathrm{B}-\mathrm{V}) \simeq 1$.2. Subsequent spectro-photometric observations in the infrared by Rudy, Puetter \& Mazuk (1999) revealed an in situ reddening of $\mathrm{E}(\mathrm{B}-\mathrm{V})=0.77 \pm 0.09$ to be added to the Galactic one of $\mathrm{E}(\mathrm{B}-\mathrm{V})=0.57$ estimated through the COBE/IRAS infrared maps ${ }^{1}$ of Schlegel, Finkbeiner \& Davis (1998).

3C 109 is also a bright source in the X-ray band with a 2$10 \mathrm{keV}$ intrinsic luminosity of $2-5 \times 10^{45} \mathrm{erg} \mathrm{s}^{-1}$ (Allen \& Fabian 1992; Allen et al 1997) making it one of the most X-ray luminous objects at $z<0.5$. Absorption is seen in the X-rays in excess of that expected from Galactic material, confirming the optical and infrared studies. ROSAT PSPC (Allen \& Fabian 1992) and ASCA (Allen et al 1997) data were used to infer a total X-ray column density of $\mathrm{N}_{H} \sim 7 \times 10^{21} \mathrm{~cm}^{-2}$, in good agreement with the optical reddening $\left(\mathrm{N}_{H}=E(B-V) \cdot 5.8 \times 10^{21} \simeq 7.7 \times 10^{21}\right.$; Bohlin, Savage \& Drage 1978). The ASCA data also revealed the presence of a broad iron (Fe) $\mathrm{K} \alpha$ emission line (Allen et al 1997). The line was consistent with being produced in the accretion disc but the emitting region was only poorly constrained with an upper limit on the inner disc radius of $\sim 140$ gravitational radii. When interpreted as due to reflection from the accretion disc, the broad Fe line profile suggested an observer inclination $\theta>35^{\circ}$ (for a neutral $6.4 \mathrm{keV}$ Fe line).

At radio wavelengths, 3C 109 is classified as a FR II lobedominated radio galaxy with steep spectrum (Laing, Riley \& Longair 1983) and large luminosity $\left(\sim 9 \times 10^{26} \mathrm{~W} \mathrm{~Hz}^{-1}\right.$; Bennet et al. 1986). The large-scale radio structure of 3C 109 consists of two symmetric lobes with hot spots and core emission. A faint and clearly one-sided jet is present at the parsec scale (Giovannini et al 1994). By measuring the jet/core flux ratio in VLA data Giovannini et al. (1994) could derive a value of $\theta<34^{\circ}$ for the angle between the radio axis and the line of sight. VLBI observations of the jet/counter-jet ratio however indicate a less tight constraint of $\theta<56^{\circ}$. By combining the radio constraints on the orientation with that from the broad $\mathrm{Fe} \mathrm{K}$ line $\left(\theta>35^{\circ}\right)$, 3C 109 seems to lie at the high-inclination end of the quasar class, which could also explain the observed excess absorption in terms of a line of sight passing through the edge of the obscuring torus.

The ASCA detection of the broad Fe line in 3C 109 represents the exception rather than the rule for radio-loud objects (e.g. Eracleous \& Halpern 1998; Woźniak et al. 1998; Eracleous, Sambruna \& Mushotzky 2000; Grandi et al. 2006). The weak disc reflection signatures in radio-loud sources possibly indicate that the $\mathrm{X}$-ray continuum is beamed away from the reflector (e.g. Beloborodov 1999) or the presence of a truncated accretion disc geometry in which the inner regions of the standard radiatively efficient and geometrically thin disc are replaced by a thick and radiatively inefficient medium such as in an advection-dominated accretion flow (Narayan \& Li 1995; see also Meier 1999; 2001 for the connec-

\footnotetext{
1 As pointed out by Rudy, Puetter \& Mazuk (1999) the COBE/IRAS estimate is much larger that the value $\mathrm{E}(\mathrm{B}-\mathrm{V})=0.27 \pm 0.03$ derived from the H I measurements of Burstein \& Heiles (1982) but should be more accurate since it is based on direct observation of dust.
}

tion with jets). An alternative explanation is that the an untruncated disc is present, but highly ionized, thus producing weaker reflection signatures than expected in the neutral case (Ballantyne et al. 2002). As mentioned, the ASCA result on 3C 109 is consistent with both an untruncated disc and one in which the innermost 150 gravitational radii are missing thereby not providing a clear picture of the innermost accretion flow, while the relatively steep $\mathrm{X}$-ray slope ( $\Gamma \simeq 1.86$ ) was used to exclude a strong contribution from a beamed jet component. So far, XMM-Newton observations of BLRGs have produced inconclusive results (e.g. Gliozzi et al. 2004; Ballantyne et al. 2004; Lewis et al. 2005) with some exceptions such as e.g. the BLRG 4C 74.26 and the radio-loud quasar PG 1425+267 in which evidence for an untruncated disc has been recently reported by Ballantyne \& Fabian (2005) and Miniutti \& Fabian (2006), respectively.

Here we present results from a new $\sim 40$ ks X-ray observation of 3C 109 with XMM-Newton. The higher sensitivity of the XMMNewton CCD detectors with respect to any other X-ray mission allows us to study the Fe band with unprecedented detail in search for a confirmation of the ASCA detection of a broad Fe emission line. We also present results on the excess absorption seen at low energies with the aim of providing a self-consistent picture of the environment of 3C 109 in the X-ray, optical, and infrared bands.

\section{THE XMM-NEWTON OBSERVATION}

3C 109 was observed by XMM-Newton on 2005 February 3-4 during revolution 944 . Source/background light curves and spectra for the EPIC detectors were extracted from circular regions centred on the source and close to it respectively. High background is present in the last few ks of the observation which are excluded from the analysis. Another period of relatively high background is instead included after verifying that it does not affect the spectra in any noticeable way. After filtering, the net exposure is $\sim 34 \mathrm{ks}$ for the pn and $\sim 37 \mathrm{ks}$ for both MOS cameras. The pn spectrum was grouped as to have a minimum of 50 counts per bin, while the MOS spectra were grouped to have 25 counts per bin.

It should be stressed that $\mathrm{X}$-ray emission from the radio lobes has been detected with Chandra (Croston et al 2005). The XMMNewton spatial resolution does not allow us to distinguish between core and lobe X-ray emission. However, Croston et al. (2005) could measure an upper limit of $3.7 \mathrm{nJy}$ for the $1 \mathrm{keV}$ flux density due to the lobes in 3C 109, while the $1 \mathrm{keV}$ flux density of the core is $896_{-109}^{+129}$ nJy (Hardcastle, Evans \& Croston 2006). Given that the $\mathrm{X}$-ray core is slightly brighter during the present XMM-newton observation than in the previous Chandra one (see Fig. 6 below), the lobe contribution to the XMM-Newton spectrum is likely limited to less than $0.3-0.4$ per cent, making the spectrum analysed below completely dominated by the core emission.

In Fig. 1, we show the EPIC pn light curve in a soft (S: $0.2-$ $2 \mathrm{keV}$, top panel) and in a hard $(\mathrm{H}: 2-10 \mathrm{keV}$, middle panel) energy band together with the resulting hardness ratio $(\mathrm{H} / \mathrm{S}$, bottom panel). The two light curves are consistent with being constant over the XMM-Newton exposure. A constant fit to the hardness ratio is also totally acceptable ( $\chi^{2}=53.5$ for 70 degrees of freedom) excluding any strong spectral variation during the observation. Since 3C 109 does not exhibit any sign of short-timescale flux or spectral variability, we concentrate on the time-averaged $\mathrm{X}$-ray spectrum of the source. 


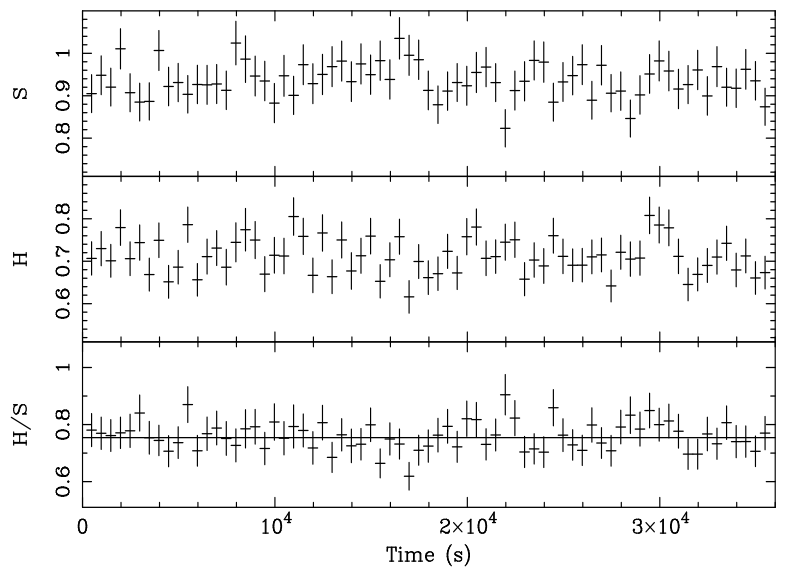

Figure 1. We show the background-subtracted EPIC pn light curves in a soft (S: $0.2-2 \mathrm{keV})$ and a hard $(\mathrm{H}: 2-10 \mathrm{keV})$ band and the resulting hardness ratio $(\mathrm{H} / \mathrm{S})$. Each time-bin is $500 \mathrm{~s}$ long. Both light curves exhibit very small amplitude variations (at the few per cent level) but a fit with a constant is acceptable for both the $\mathrm{S}$ and the $\mathrm{H}$ bands. In the bottom panel, we also show the best-fit constant model for the harness ratio $(\mathrm{H} / \mathrm{S}=0.754 \pm 0.006)$ which results in a $\chi^{2}=53.5$ for 70 degrees of freedom.

\section{THE 2-10 KEV SPECTRUM AND FE K BAND}

We start our analysis by considering the hard spectrum of 3C 109 in the $2-10 \mathrm{keV}$ band (observed frame, i.e. $2.6-13.06 \mathrm{keV}$ in the rest-frame, $\mathrm{z}=0.3056$ ). We consider the EPIC-pn data only because of the higher sensitivity of the pn camera with respect to MOS in the Fe band. A simple absorbed power law model provides a good description of the hard spectrum for a power law photon index of $\Gamma=1.67_{-0.05}^{+0.07}$. Neutral photoelectric absorption is also seen even above $2 \mathrm{keV}$ and we measure a column density of $N_{H}=3.5_{-1.7}^{+2.5} \times$ $10^{21} \mathrm{~cm}^{-2}$. The actual value of the column density will be better constrained by including the soft data, and we defer any discussion to a further analysis below. The overall fit is acceptable with $\chi^{2}=$ 367 for 398 degrees of freedom (dof), but visual inspection of the residuals suggests the possible presence of emission features in the Fe K band. In Fig. 2 we show the data to model ratio in the Fe K band for the simple model above. The vertical line marks the energy of $6.4 \mathrm{keV}(\mathrm{Fe} \mathrm{K} \alpha)$ in the source rest-frame.

\subsection{Fe emission: simple models}

To account for the residuals in Fig. 2, we add to the model a Gaussian emission line and find a significant improvement of $\Delta \chi^{2}=14$ for three more free parameters corresponding to a significance at the $\sim 99.9$ per cent level according to the F-test for a final result of $\chi^{2}=353$ for 395 dof. However, as pointed out by Protassov et al. (2002), the estimate of the statistical significance of emission lines through the F-test is not highly accurate. We then performed Monte Carlo simulations according to the method proposed in Porquet et al. (2004) and our results confirm the significance of the Fe line detection at more than the 99.6 per cent confidence level. The bestfitting parameters indicate a line energy of $E=6.62 \pm 0.18 \mathrm{keV}$ with a rest-frame equivalent width $\mathrm{EW}=90 \pm 35 \mathrm{eV}$ with respect to the absorbed power law continuum. The line appears to be barely resolved and we measure a width $\sigma=220 \pm 170 \mathrm{eV}$.

However, Fig. 2 suggests the presence of a more structured line profile and the possible presence of two emission lines. The brightest is close to $6.4 \mathrm{keV}$, while the fainter is seen at higher en- ergy. As a first phenomenological model, we consider a doubleGaussian parametrisation of the emission features. We obtain a slightly better fit than with a single Gaussian $\left(\chi^{2}=348\right.$ for 392 dof). One line is found at $E=6.46_{-0.05}^{+0.09} \mathrm{keV}$ and has an equivalent width of $60 \mathrm{eV}$ in the rest-frame. The second line is at $E=6.86_{-0.23}^{+0.11} \mathrm{keV}$ and is fainter. Both lines are unresolved with an upper limit on their width of $200 \mathrm{eV}$ and $300 \mathrm{eV}$ respectively. The energy of the lines indicates the presence of a slightly ionized reflection component producing a $\sim 6.4 \mathrm{keV}$ neutral $\mathrm{K} \alpha$ line and a fainter $\mathrm{He}-$ like one.

\subsubsection{A relativistic Fe line}

A second parametrisation is suggested by previous X-ray observations of $3 \mathrm{C} 109$. An Fe emission line was first detected in the X-ray spectrum of 3C 109 with ASCA and result are reported by Allen et al (1997). When fitted with a single Gaussian emission line, the XMM-Newton best-fitting parameters (see above) are different but consistent within the 90 per cent errors with the ASCA results. In particular a much larger width $\left(\sigma=650_{-360}^{+810} \mathrm{eV}\right)$ and equivalent width $\left(300_{-200}^{+600} \mathrm{eV}\right)$ were suggested by the $A S C A$ spectrum. The Fe line detected by ASCA was broad and consistent with being emitted from the accretion disc. The Fe line emitting region was however poorly constrained and the inner disc radius was allowed to take any value from the innermost stable circular orbit (ISCO) around a non-rotating black hole up to $140 r_{g}\left(r_{g}=G M / c^{2}\right.$ : the ISCO for a non-rotating black hole is $6 r_{g}$ ).

To directly compare the $X M M-N e w t o n$ data with the ASCA results on the Fe line, we replace the Gaussian emission line with a DISKLINE model (Fabian et al 1989) as in Allen et al (1997). We fix the line energy to $6.4 \mathrm{keV}$ and the emissivity to the energy dissipation profile of a standard accretion disc $\left(r^{-3}\right)$. The inner disc radius and observer inclination are free to vary while the outer disc radius is fixed to $10^{3} r_{g}$. We obtain a good fit with the same statistical quality as the one with a Gaussian line (model A in Table 1). The inner disc radius can take any value between the ISCO around a non-rotating black hole and $27 r_{g}$, significantly improving the ASCA $140 r_{g}$ constraint, while the observer inclination is $i=43^{\circ} \pm 12^{\circ}$. If the line energy is instead fixed to be $6.7 \mathrm{keV}$, corresponding to $\mathrm{He}-$ like $\mathrm{Fe}$ emission, only a lower limit on the inclination can be obtained $\left(i>34^{\circ}\right)$ and the inner disc radius is completely unconstrained $\left(6 r_{g}<r_{\text {in }}<855 r_{g}\right)$. Results are thus not conclusive on the presence of a relativistic Fe line in 3C 109. The data are consistent with a relativistic $\mathrm{Fe}$ line from a disc extending all the way down to the ISCO, but a truncated disc missing the innermost 20-30 $r_{g}$ gravitational radii cannot be excluded.

The Fe line EW is smaller than in the ASCA data (see Table 1 , model A), but the line intensity is consistent with being constant within the errors. By considering the neutral $(6.4 \mathrm{keV})$ $\mathrm{Fe}$ line model, we measure an intensity of $(1.3 \pm 0.6) \times$ $10^{-5} \mathrm{ph} \mathrm{cm}^{-2} \mathrm{~s}^{-1}$ to be compared with the ASCA result of $\left(2.1_{-1.3}^{+4.3} \times 10^{-5} \mathrm{ph} \mathrm{cm}^{-2} \mathrm{~s}^{-1}\right.$.

\subsection{Including the $\mathrm{X}$-ray reflection continuum}

If the Fe line really originates in optically thick matter such as the accretion disc (or the torus if narrow), the line is associated with a reflection continuum that has to be included in the spectral model for self-consistency. The $\mathrm{X}$-ray reflection continuum introduces curvature (and Fe edge) in the Fe band and could affect the line parameters. Moreover, when the reflection spectrum 


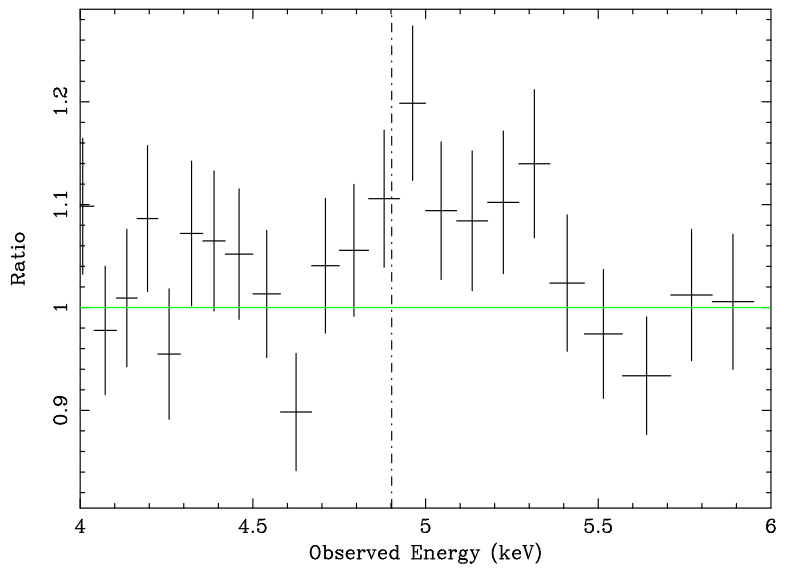

Figure 2. Data to model ratio in the Fe $\mathrm{K}$ band. The model is a simple absorber power law fitted in the $2-10 \mathrm{keV}$ band ignoring the Fe band. Data have been rebinned for visual clarity. The vertical line is at $6.4 \mathrm{keV}$ in the source rest-frame.

is ionized, Comptonization induces a broadening of the line which has to be taken into account. We then apply to the hard spectrum a self-consistent ionized reflection model in which the emission lines are computed together with the X-ray reflection continuum (Ross \& Fabian 2005). The model parameters are the Fe abundance and source redshift (that we fix to the solar value and to the source redshift respectively), the ionization parameter of the reflector, the photon index of an incident power law continuum (which has a high-energy cutoff at $100 \mathrm{keV}$ ) and the normalisation.

\subsubsection{Reflection from distant matter}

As a first step, we assume that the reflection spectrum and Fe features come from distant material so that the only Fe line broadening can be due to Comptonization if the reflector is highly ionized. We obtain a good fit to the $2-10 \mathrm{keV}$ pn spectrum (model $\mathrm{B}$ in Table 1). The ionization parameter is constrained to be smaller than $244 \mathrm{erg}^{\mathrm{cm} \mathrm{s}}{ }^{-1}$. The best-fitting reflection spectrum comprises both a neutral $6.4 \mathrm{keV}$ line and a fainter He-like one at $6.7 \mathrm{keV}$. The data to model ratio in the Fe band is shown in the top panel of Fig. 3. Residuals are left above $6.4 \mathrm{keV}$ (rest-frame, vertical line) and we tried to account for them by adding a second more ionized reflector, but we did not find any improvement in the statistics. We measure a reflection fraction of $0.8 \pm 0.2$, i.e. the reflector intercepts about half (or only slightly less) of the X-ray continuum in good agreement also with the measured $\mathrm{Fe}$ line equivalent width (see model A in Table 1).

\subsubsection{Accretion disc reflection}

We then consider the same reflection model as above, but we apply a relativistic blurring kernel (obtained from the DISKLINE model) inducing the relativistic effects arising if emission comes from an accretion disc around a non-rotating black hole (model $\mathrm{C} 1$ in Table 1). As for model A, we fix the emissivity to its standard profile $r^{-3}$. We obtain an improvement of $\Delta \chi^{2}=7$ with respect to the unblurred reflection model $\mathrm{B}$ for two more free parameters which means that the relativistic blurring is significant at the 98 per cent level. The inner disc radius is constrained to be smaller than $26 r_{g}$, while the observer inclination is measured to be $i=40^{\circ} \pm 8^{\circ}$, confirming the results obtained with the simpler power law plus

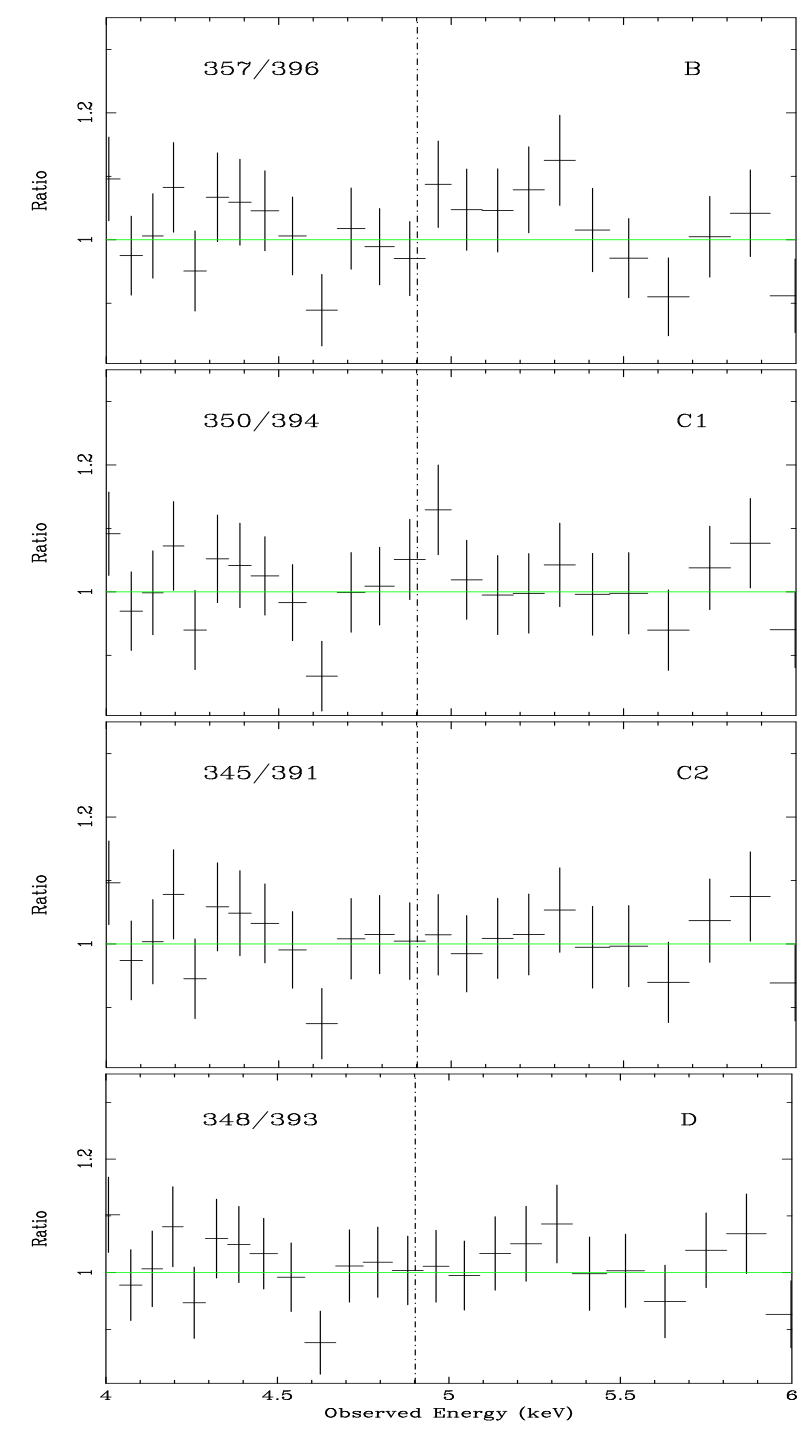

Figure 3. We show the data to model ratio in the Fe $\mathrm{K}$ band for the different models used to describe the $2-10 \mathrm{keV}$ spectrum of $3 \mathrm{C} 109$ (see text and Table 1 for details). From top to bottom models B, C1, C2, and D are shown together with the resulting statistics ( $\left.\chi^{2} / \mathrm{dof}\right)$. The vertical line is at $6.4 \mathrm{keV}$ in the source rest-frame.

DISKLINE model A when the line was assumed to be neutral. As for the ionization state, we measure a low ionization parameter $\xi<30 \mathrm{erg} \mathrm{cm} \mathrm{s}^{-1}$ which again points towards a neutral (or slightly ionized) accretion disc. We also tried to artificially increase the ionization of the reflector to reproduce the results obtained with model A when the line energy was fixed to $6.7 \mathrm{keV}$ but we could not find any solution. Once again, the best-fitting solution points toward a significant contribution of the reflection component (with a reflection fraction of the order of unity) suggesting a typical Seyfert 1like X-ray continuum.

\subsubsection{Composite reflection (disc plus distant matter)}

Visual inspection of the residuals shows that the $6.4 \mathrm{keV}$ peak is not well modelled (second panel of Fig. 3). We then add to model $\mathrm{C} 1$ a narrow Gaussian emission line representing the narrow component of the Fe $\mathrm{K}$ line (model $\mathrm{C} 2$ in Table 1 ). A narrow Fe $\mathrm{K}$ line is in fact ubiquitous in the $\mathrm{X}$-ray spectra of Active galaxies and 


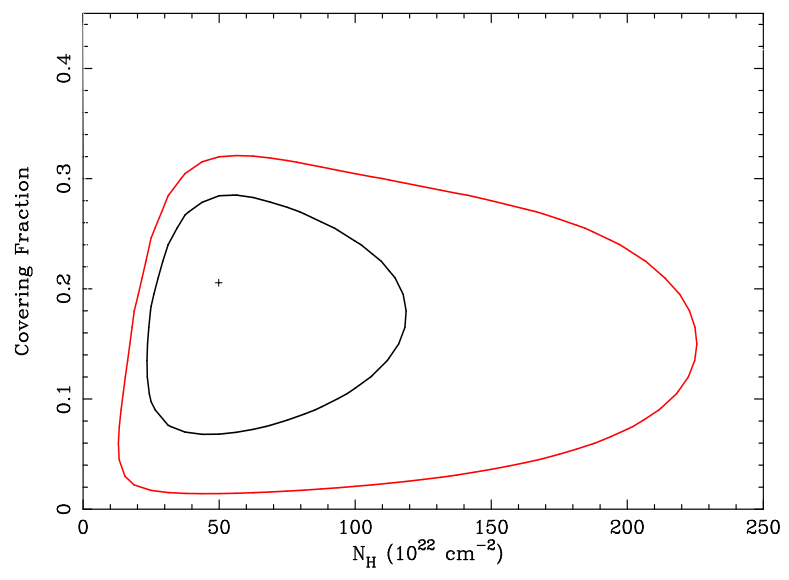

Figure 4. Confidence level contours (68 and 90 per cent) for the covering fraction and the column density of the partial covering model to the $2-$ $10 \mathrm{keV}$ spectrum (model D).

is thought to be associated with some distant reflector such as the torus of unification schemes (e.g. Yaqoob \& Padmanabhan 2004). The inclusion of the narrow emission line marginally improves the fit by $\chi^{2}=5$ for three more free parameters. The line parameters are reported in Table 1 and the data to model ratio is shown in the third panel of Fig. 3. The blurring reflector parameters (inner disc radius and inclination) are fitted to the data being however unaffected and indistinguishable from results obtained with model $\mathrm{C} 1$. If the narrow line is replaced by an unblurred reflection spectrum no further improvement is obtained.

\subsection{Partial covering alternative to the relativistic Fe line}

The $2-10 \mathrm{keV}$ spectrum is best fitted (though marginally) by model $\mathrm{C} 2$ comprising an almost neutral reflection spectrum from the accretion disc plus a component from distant matter producing the narrow $\mathrm{Fe}$ line at $6.4 \mathrm{keV}$. As mentioned, if relativistic blurring is removed the statistical quality of the fit is only slightly worse and we are thus motivated to find alternative solutions. Here we briefly explore one further alternative to the relativistic line.

We consider the possibility that absorption by a large column of gas partially covering the source is responsible for the hard spectral shape, as tentatively observed in other radio-loud sources (e.g. Lewis et al. 2005). We also include a Gaussian emission line to account for possible $\mathrm{Fe}$ emission from the absorber itself (or other distant material). We obtain a good fit with a partial covering model (model D) which is statistically indistinguishable from our bestfitting model C2 (see Table 1). The data to model ratio for model D are shown in the bottom panel of Fig. 3. The partial covering parameters are however not very well constrained. The covering fraction is only an upper limit of 32 per cent and the column density can take any value from $10^{23} \mathrm{~cm}^{-2}$ to $2.3 \times 10^{24} \mathrm{~cm}^{-2}$ ( $2 \sigma$ errors). The 68 and 90 per cent confidence level contours for covering fraction and column density are shown in Fig. 4. We also replaced the neutral partial coverer with an ionized one, but the requirement is for neutral matter with a 90 per cent upper limit on the ionization parameter of $\xi<0.5 \mathrm{erg} \mathrm{cm} \mathrm{s}^{-1}$.

\subsection{Conclusions and estimate of the Eddington ratio}

We conclude that the spectral shape in the hard band is well reproduced by either $i$ ) an almost neutral $\mathrm{X}$-ray reflection spectrum from

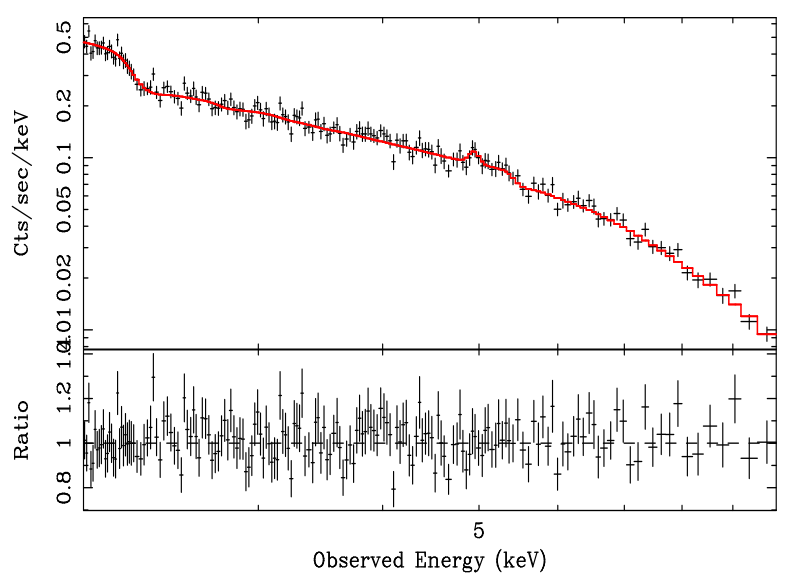

Figure 5. We show the $2-10 \mathrm{keV}$ pn spectrum and data to model ratio for model C2 (see text and Table 1).

the accretion disc with a likely contribution from a distant reflector (model $\mathrm{C} 2$ ) or by ii) intervening absorption by a large column of gas $\left(>10^{23} \mathrm{~cm}^{-2}\right.$ ) covering about 20 per cent of the X-ray continuum source plus a narrow Fe $\mathrm{K} \alpha$ line (model D). Both interpretations are allowed by the data and cannot be distinguished on a statistical basis. In Fig. 5 we show the $2-10 \mathrm{keV}$ spectrum and data to model ratio when the spectrum is fitted with model $\mathrm{C} 2$.

By choosing $\mathrm{C} 2$ as best-fit model, we measure an observed 2-10 keV flux of $6.8 \times 10^{-12} \mathrm{erg} \mathrm{cm}^{-2} \mathrm{~s}^{-1}$. Correcting for absorption, we have a $2-10 \mathrm{keV}$ flux of $7 \times 10^{-12} \mathrm{erg} \mathrm{cm}^{-2} \mathrm{~s}^{-1}$, corresponding to a $2-10 \mathrm{keV}$ luminosity of $2 \times 10^{45} \mathrm{erg} \mathrm{s}^{-1}$ in the source rest-frame. If partial covering is instead the right description of the hard spectrum, the intrinsic absorption corrected luminosity is $\simeq 3 \times 10^{45} \mathrm{erg} \mathrm{s}^{-1}$, depending on the assumed column density $\left(H_{0}=70 \mathrm{~km} \mathrm{~s}^{-1} \mathrm{Mpc}^{-1}, \Omega_{\Lambda}=0.73, \Omega_{0}=1\right)$.

Given the recent mass estimate for the central black hole ( $M_{\mathrm{BH}} \simeq 2 \times 10^{8} M_{\odot}$, McLure et al 2006), and by assuming a 2$10 \mathrm{keV}$ to bolometric luminosity conversion ${ }^{2}$ of a factor 30 (Fabian \& Iwasawa 1999 from the compilation of spectral energy distributions of Elvis et al. 1994), it appears that 3C 109 is characterised by a super-Eddington luminosity with $L_{\mathrm{bol}} / L_{\mathrm{Edd}} \simeq 2.5$. The black hole mass estimate is crucial for this result: McLure et al. (2005) used the $\mathrm{H} \beta$ version of the virial-mass estimator (McLure \& Dunlop 2004) which provides black hole mass estimates with a known scatter of $\sim 0.4$ dex, meaning that the black hole mass of 3C 109 could be as large as $\sim 5 \times 10^{8} M_{\odot}$. However, even by adopting this upper limit, the requirement is still for a source radiating at its Eddington luminosity.

The above discussion assumes that the $\mathrm{X}$-ray luminosity is radiated isotropically and, given the FR II nature of 3C 109, this is far from obvious. It is indeed possible that part of the $\mathrm{X}$-ray core emission is due to a beamed inner jet component, which would reduce the $L_{\text {bol }} / L_{\text {Edd }}$ ratio. The jet component is generally associated with a flatter (1.5-1.7) X-ray slope than measured here (1.86). However, it should be stressed that some of the knots in the jets of FR II radio galaxies can have much steeper photon-indexes than

2 The adopted bolometric correction of 30 for the $2-10 \mathrm{keV}$ luminosity is a conservative one and is likely to underestimate the actual bolometric luminosity of 3C 109 by a factor 2-3, see e.g. Elvis, Risaliti \& Zamorani 2002. Therefore the Eddington ratio discussed below has to be intended as a lower limit. 
Table 1. Summary of the best-fit parameters and 90 per cent errors $\left(\Delta \chi^{2}=2.71\right.$ for one parameter) for the most relevant spectral models used to describe the X-ray spectrum of 3C 109. Only the EPIC-pn data are used in the $2-10 \mathrm{keV}$ band, while the MOS 1 and MOS 2 data are included in the broadband $(0.4-10 \mathrm{keV})$ analysis. The parameters refer to the $2-10 \mathrm{keV}$ analysis but consistent results (within the errors) are obtained when the broadband spectrum is considered except for the photon index of model A that steepens to $\Gamma \simeq 1.75$ in the $0.4-10 \mathrm{keV}$ analysis.

\begin{tabular}{|c|c|c|c|c|c|c|c|c|}
\hline MODEL & \multicolumn{6}{|c|}{ PARAMETERS } & \multirow{2}{*}{$\begin{array}{c}2-10 \mathrm{keV} \\
\chi^{2} / \mathrm{dof}\end{array}$} & \multirow{2}{*}{$\begin{array}{c}0.4-10 \mathrm{keV} \\
\chi^{2} / \mathrm{dof}\end{array}$} \\
\hline \multirow{3}{*}{$\begin{array}{c}\text { A } \\
\text { POW + DISKLINE }\end{array}$} & $\Gamma$ & $\mathrm{E}_{\mathrm{Fe}}$ & $r_{\text {in }}$ & $\mathrm{i}$ & $\mathrm{EW}_{\mathrm{Fe}}$ & - & & \\
\hline & $1.68 \pm 0.07$ & $6.4^{f}$ & $<27 r_{g}$ & $43^{\circ} \pm 12^{\circ}$ & $170 \pm 70$ & - & $353 / 395$ & $1322 / 1364$ \\
\hline & $1.68 \pm 0.07$ & $6.7^{f}$ & $380_{-374}^{+475} r_{g}$ & $>34^{\circ}$ & $105 \pm 40$ & - & $351 / 395$ & $1319 / 1364$ \\
\hline B & $\Gamma$ & - & - & - & $\xi$ & $\mathrm{R}$ & & \\
\hline POW + REF & $1.75_{-0.08}^{+0.06}$ & - & - & - & $<244$ & $0.8 \pm 0.2$ & $357 / 396$ & $1317 / 1365$ \\
\hline $\mathrm{C} 1$ & $\Gamma$ & - & $r_{\text {in }}$ & $\mathrm{i}$ & $\xi$ & $\mathrm{R}$ & & \\
\hline POW + DISCREF & $1.87 \pm 0.10$ & - & $<26 r_{g}$ & $40^{\circ} \pm 8^{\circ}$ & $<30$ & $1.1 \pm 0.2$ & $350 / 394$ & $1297 / 1363$ \\
\hline $\mathrm{C} 2$ & $\Gamma$ & $\mathrm{E}_{\mathrm{Fe}}$ & $\sigma$ & $\mathrm{EW}_{\mathrm{Fe}}$ & $\xi$ & $\mathrm{R}$ & & \\
\hline POW + DISCREF + GAUSS & $1.87 \pm 0.10$ & $6.45_{-0.06}^{+0.09}$ & $<340$ & $45 \pm 15$ & $<45$ & $1.1 \pm 0.2$ & $345 / 391$ & $1291 / 1360$ \\
\hline $\mathrm{D}$ & $\Gamma$ & $\mathrm{E}_{\mathrm{Fe}}$ & $\sigma$ & $\mathrm{EW}_{\mathrm{Fe}}$ & $\mathrm{N}_{\mathrm{H}}$ & $\mathrm{f}_{\mathrm{c}}$ & & \\
\hline PC ( POW + GAUSS ) & $1.86 \pm 0.08$ & $6.47_{-0.05}^{+0.14}$ & $<325$ & $50_{-30}^{+60}$ & $500_{-390}^{+1800}$ & $<32 \%$ & $348 / 393$ & $1292 / 1362$ \\
\hline
\end{tabular}

All fits include photoelectric absorption (the WABS model). The resulting column density is not reported here. It is always of the order of $3-5 \times 10^{21} \mathrm{~cm}^{-2}$ and a detailed analysis of the absorption in $3 \mathrm{C} 109$ is given below (see Section 5 and Table 2). In models $\mathrm{A}$ and $\mathrm{D}$ the $\mathrm{Fe}$ line energy $\mathrm{E}_{\mathrm{Fe}}$ is given in keV in the source rest-frame, while the line width and equivalent width (EW) are given in $\mathrm{eV}$ (the $\mathrm{EW}$ is also corrected for the source redshift). In model $\mathrm{B}, \mathrm{C} 1$, and $\mathrm{C} 2$ REF refers to the unblurred reflection model, and DISCREF to the relativistically blurred one. The ionization parameter of the reflection models $(\xi)$ is given in units of erg cm s$~^{-1}$ and $\mathrm{R}$ is the reflection fraction ( $\mathrm{R}=1$ means that the reflector intercepts about half of the $\mathrm{X}$-ray continuum). Given that our reflection model does not contain the reflection fraction as a free parameter, its value is estimated by comparison of the best-fitting reflection model with the PEXRAV model (neutral reflection), a procedure that is reasonably accurate here given that the ionization state of the reflector is low. In model C2 the parameters $r_{\text {in }}$ and $i$ are the same as in model $\mathrm{C} 1$ and are not reported. In model D, the column density of the partial coverer is given in units of $10^{21} \mathrm{~cm}^{-2}$.

generally seen $(\Gamma \sim 2$ though with large error bars, see e.g. Sambruna et al. 2006). Thus the steep photon index we measure cannot be taken as a secure indication that beamed emission is negligible. On the other hand, if reflection rather than partial covering is the rigth description of the hard $\mathrm{X}$-ray data, the measured reflection fraction (of the order of unity) implies that the X-ray continuum is not strongly beamed away from the reflector, thus suggesting little inner jet contamination. We conclude that, though it is difficult to exclude that the inferred bolometric luminosity is over-estimated because of the presence of a beamed component, the possibility that $3 \mathrm{c} 109$ is radiating a substantial fraction (close to unity) of its Eddington luminosity does remain plausible, which would also be consistent with the tentative detection of the broad Fe line from the accretion disc.

\section{BROADBAND SPECTRAL ANALYSIS}

We then consider the broadband spectrum of 3C 109. We use the full 0.4-10 keV band and consider joint fits to all EPIC cameras (pn and the two MOS). The data below $0.4 \mathrm{keV}$ are ignored because of some residual inter-calibration problems at the softest energies. The extension of the $2-10 \mathrm{keV}$ spectral models to the soft band is satisfactory (even before fitting the data) for all models. In the last column of Table 1 we report the results of the fits to the broadband spectrum with the same models used for the hard band. The parameters reported in Table 1 refer to the $2-10 \mathrm{keV}$ fits but are basically unaffected by the inclusion of the low energy data except for the photon index of models A which steepens to $\Gamma \simeq 1.75$.
Once again the data tend to prefer models $\mathrm{C} 2$ and $\mathrm{D}$ as the best-fitting solutions which are indistinguishable from a statistical point of view. We notice that models $\mathrm{C} 1, \mathrm{C} 2$, and $\mathrm{D}$ have a steeper spectral index than models $\mathrm{A}$ and $\mathrm{B}$. This is the main reasons for the better fits provided by models $\mathrm{C} 1, \mathrm{C} 2$ and $\mathrm{D}$. In fact if only the soft data below $2.5 \mathrm{keV}$ are considered (where the reflection/partial covering contribution is negligible) a fit with an absorbed power law gives $\Gamma \simeq 1.85$ confirming that a steep photon index is preferred by the data as in models $\mathrm{C} 1, \mathrm{C} 2$, and $\mathrm{D}$ and suggesting that models $\mathrm{A}$ and $\mathrm{B}$ have to be considered as less likely as also indicated by the fitting statistics.

\subsection{Long-term X-ray variability}

The broadband spectral analysis is in excellent agreement with the ASCA data presented in Allen et al. (1997) and enable us to compare the observed flux with previous X-ray observations. We consider the $1-2 \mathrm{keV}$ flux only, since this energy band is common to all previous observations. We measure a flux of $(14.5 \pm 0.3) \times 10^{-13}$ and, in Fig. 6, we show the historical X-ray flux light curve in that same energy band. All historical data are re-collected from Allen et al (1997) with the exception of a second 1998 ASCA observation (unpublished) and of the 2003 Chandra observation (Hardcastle, Evans \& Croston 2006) that we include here for the first time. Long-term X-ray variability is clearly present with the largest amplitude variation occurring in a 4 years interval between the 1991 ROSAT and the 1995 ASCA observations (about a factor 2). Variability with the similar amplitude and timescale has also been reported in the near-infrared (Rudy et al. 1984) and variation of the 


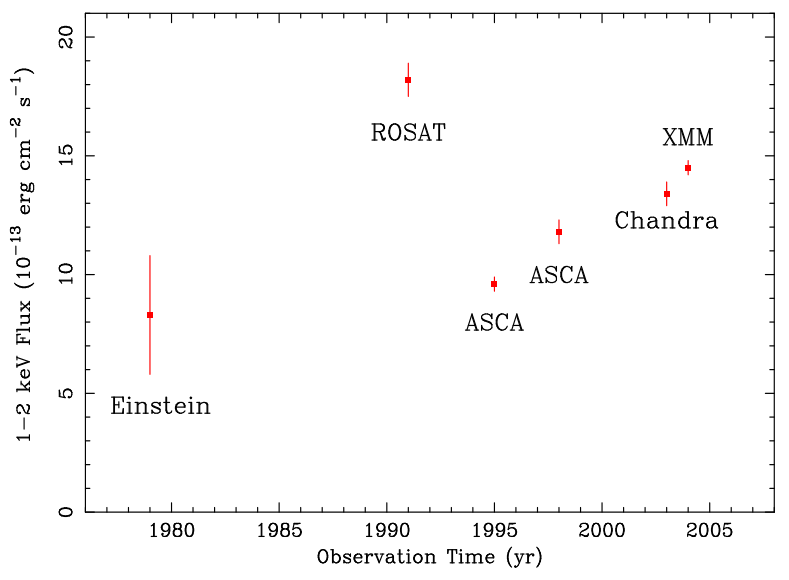

Figure 6. The historical X-ray flux of 3C 109 in the 1-2 keV band.

order of 50 per cent are seen in the $\mathrm{J}, \mathrm{H}$, and $\mathrm{K}$ bands as well (Elvis et al. 1984).

\section{EXCESS ABSORPTION}

When model $\mathrm{D}$ is extended to the $0.4-10 \mathrm{keV}$ band, we measure (besides the partial covering column density) a column density of $\mathrm{N}_{\mathrm{H}}=4.64_{-0.21}^{+0.09} \times 10^{21} \mathrm{~cm}^{-2}$ which is derived by assuming that the absorber lies at zero redshift. An identical result is obtained by using model C2 instead.

The value of the Galactic column density in the line of sight towards 3C 109 is somewhat ambiguous. The nominal value obtained through $\mathrm{H}$ I measurements is $1.57 \times 10^{21} \mathrm{~cm}^{-2}$ (Burstein \& Heiles 1982). However, COBE/IRAS maps indicate a Galactic column as high as $3.31 \times 10^{21} \mathrm{~cm}^{-2}$ (Schlegel, Finkbeiner \& Davis 1998), in good agreement with $X$-ray studies of the nearby cluster Abell 478 suggesting a column of $\sim 2.5-3 \times 10^{21} \mathrm{~cm}^{-2}$ (Sun et al. 2003; Pointecouteau 2004; Sanderson, Finoguenov \& Mohr 2005). The same result is obtained by averaging the COBE/IRAS reddening over a $1 \mathrm{deg}^{2}$ region centred on 3C 109 and, since this result is based on direct observations of the dust, we adopt this value as already done by Rudy, Puetter \& Mazuk (1999) in their infrared study in which the total column density towards 3C 109 was estimated to be $\sim 7.7 \times 10^{21} \mathrm{~cm}^{-2}$.

The column density we measure in the broadband fits $\left(4.64_{-0.21}^{+0.09} \times 10^{21} \mathrm{~cm}^{-2}\right)$ strongly suggests that excess absorption is present, confirming previous analysis of ROSAT PSPC and ASCA data (Allen \& Fabian 1992; Allen et al 1997). Assuming, as we did so far, that the absorber lies at zero redshift, a column density of $(5.30 \pm 0.42) \times 10^{21} \mathrm{~cm}^{-2}$ was inferred from previous ASCA spectral analysis, while ROSAT data indicates a total column density of $4.2_{-1.6}^{+1.9} \times 10^{21} \mathrm{~cm}^{-2}$. The XMM-Newton data confirm the presence of excess absorption in the line of sight with a (zero redshift) column density somewhat lower than the ASCA result and consistent with the ROSAT spectrum.

Since we are here interested in determining the properties of an absorber with column density of a few times $10^{21} \mathrm{~cm}^{-2}$ and given that such a column does not affect significantly the spectrum above $3 \mathrm{keV}$, in the following we consider the $0.4-2.5 \mathrm{keV}$ band only. In this way, we are not affected by the spectral complexities in the hard band (partial covering and/or reflection) and we are certain of measuring the intrinsic spectral slope, whose value clearly affects the absorption properties. In Fig. 7 we show the confidence

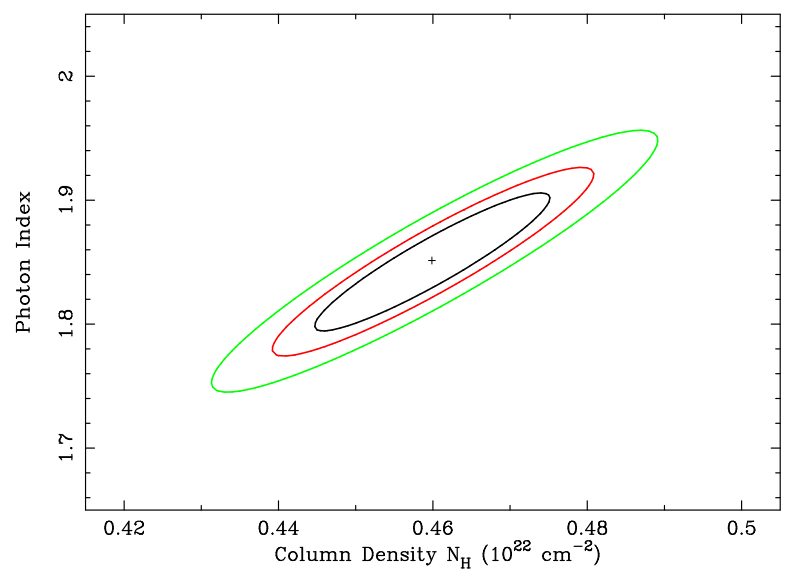

Figure 7. Confidence contours for photon index and total column density from the absorbed power law model fit to the pn and MOS data in the 0.4$2.5 \mathrm{keV}$ band (model I in Table 2). Contours represent the 68, 90, and 99 per cent confidence levels.

level contours for the power law photon index and total column density (model I in Table 2). The contours confirm that the total column density seen in the X-ray spectrum of 3C 109 is well in excess of all estimates for the Galactic value.

\subsection{Excess absorption local to 3C 109 ?}

Part of the excess absorption could be due to material surrounding the active nucleus and therefore at the redshift of $3 \mathrm{C} 109$. We first add a second neutral absorber with redshift free to vary between zero and the source redshift (0.3065). Given the uncertainties in the Galactic absorption, we make a conservative choice and we assume the highest estimated value for the Galactic column density in the line of sight $\left(3.31 \times 10^{21} \mathrm{~cm}^{-2}\right)$. We obtain a good fit but the redshift of the second absorber is only a 90 per cent upper limit $(z<0.02)$ ruling out the presence of a neutral absorber at the redshift of the source. The same result is obtained if the Galactic column density is free to vary and the second absorption component is imposed to arise at $\mathrm{z}=0.3056$ (see Table 2, model II). In this case, it is the column density of the absorber at $\mathrm{z}=0.3056$ that is only an upper limit $\left(<9 \times 10^{20} \mathrm{~cm}^{-2}\right)$.

\subsection{A "tepid absorber" solution}

The presence of excess absorption with column densities of the order of few times $10^{21} \mathrm{~cm}^{-2}$ (or more) is often detected in the Xray spectra of Active Galactic Nuclei. Given the luminosities of the central engine, the absorbing gas is almost always ionized and gives rise to typical signatures in the soft X-ray band. The soft Xray spectrum of 3C 109 does not present any clear indication that a warm absorber is present. However, if the ionization parameter of the absorbing gas is low (but different from zero) subtle differences are expected with a neutral absorber without strong signatures in the soft band.

To test the hypothesis that the excess absorption in 3C 109 is slightly ionized, we use the simple ABSORI model in XSPEC (Done et al. 1992). As in model II, we first fix the column density of the (neutral) zero-redshift absorber to the Galactic estimate and let the ionized absorber redshift be a free parameter between 0 and 0.3056 (model III in Table 2). We find a significant improvement with $\Delta \chi^{2}=12$ for one more free parameters with respect to model 
Table 2. Summary of the best-fitting parameters and 90 per cent errors for the different absorption models used to describe the $0.4-2.5 \mathrm{keV}$ spectrum of 3C 109. Data from the EPIC pn and MOS cameras are fitted jointly.

\begin{tabular}{|c|c|c|c|c|c|c|}
\hline MODEL & \multicolumn{5}{|c|}{ PARAMETERS } & \multirow[t]{2}{*}{$\chi^{2} / \mathrm{dof}$} \\
\hline I & $\Gamma$ & $\mathrm{N}_{\mathrm{H}}$ & - & - & - & \\
\hline WABS * POW & $1.85_{-0.06}^{+0.04}$ & $4.60 \pm 0.17$ & - & - & - & $613 / 632$ \\
\hline \multirow{3}{*}{$\begin{array}{c}\text { II } \\
\text { WABS } * \text { ZWABS } * \text { POW }\end{array}$} & $\Gamma$ & $\mathrm{N}_{\mathrm{H}}$ & $\mathrm{N}_{\mathrm{H}}(z)$ & $z$ & - & \\
\hline & $1.84_{-0.04}^{+0.06}$ & $3.31^{f}$ & $1.20_{-0.10}^{+0.25}$ & $<0.02$ & - & $612 / 631$ \\
\hline & $1.84 \pm 0.07$ & $4.57_{-0.58}^{+0.10}$ & $<0.9$ & $0.3056^{f}$ & - & $613 / 631$ \\
\hline \multirow{3}{*}{$\begin{array}{c}\text { III } \\
\text { WABS * ABSORI * POW }\end{array}$} & $\Gamma$ & $\mathrm{N}_{\mathrm{H}}$ & $\mathrm{N}_{\mathrm{H}}(z)$ & $z$ & $\xi_{\text {abs }}$ & \\
\hline & $1.85_{-0.06}^{+0.07}$ & $3.31^{f}$ & $3.00_{-0.40}^{+0.67}$ & $>0.28$ & $0.55_{-0.40}^{+0.25}$ & $600 / 630$ \\
\hline & $1.86_{-0.05}^{+0.07}$ & $3.50 \pm 0.55$ & $2.80 \pm 1.25$ & $0.3056^{f}$ & $0.70_{-0.35}^{+0.55}$ & $599 / 630$ \\
\hline IV & $\Gamma$ & $\mathrm{N}_{\mathrm{H}}$ & $\mathrm{N}_{\mathrm{H}}(z)$ & $z$ & $\xi_{\text {abs }}$ & \\
\hline WABS * XSTAR * POW & $1.91_{-0.06}^{+0.07}$ & $3.36_{-0.62}^{+0.41}$ & $4.00_{-1.80}^{+2.30}$ & $0.3056^{f}$ & $0.39_{-0.17}^{+0.43}$ & $588 / 630$ \\
\hline
\end{tabular}

All column densities are given in units of $10^{21} \mathrm{~cm}^{-2}$. The ionization parameter $(\xi)$ is given in units of $\mathrm{erg} \mathrm{cm} \mathrm{s}^{-1}$.

II (a significance of more than 99.95 per cent). The absorber is only slightly ionized with $\xi=0.55_{-0.40}^{+0.25} \mathrm{erg} \mathrm{cm} \mathrm{s}^{-1}$ and the 90 per cent lower limit on the redshift of the ionized absorber is $z>0.28$ indicating an in situ origin. With the ionized solution, we measure a column density of $3.00_{-0.47}^{+0.67} \times 10^{21} \mathrm{~cm}^{-2}$ in excess of the Galactic. We then remove the degeneracy between ionization parameter and redshift by fixing the redshift of the ionized absorber to $z=0.3056$ and let the Galactic column density free to vary. We find a similar fit as the one above with $N_{H}^{\mathrm{Gal}}=(3.50 \pm 0.55) \times 10^{21} \mathrm{~cm}^{-2}$ and $N_{H}^{\mathrm{Z}}=(2.80 \pm 1.25) \times 10^{21} \mathrm{~cm}^{-2}$, while the ionized absorber has a ionization parameter of $\xi=0.70_{-0.35}^{+0.55} \mathrm{erg} \mathrm{cm} \mathrm{s}^{-1}$.

As a final test, we consider a more refined absorption model using the XSTAR code (Kallman \& Bautista 2001). We find an excellent fit to the $0.4-2.5 \mathrm{keV}$ data with a significant improvement on any previous spectral model (see Table 2, model IV). Our best-fitting model is obtained by fixing the ionized absorber redshift only, while the Galactic and in situ column densities and the absorber ionization parameter are free to vary. We measure a zero-redshift, neutral column of $N_{H}^{\text {Gal }}=3.36_{-0.62}^{+0.41} \times$ $10^{21} \mathrm{~cm}^{-2}$, while the ionized absorber has a column density of $N_{H}^{\mathrm{z}}=4.00_{-1.80}^{+2.30} \times 10^{21} \mathrm{~cm}^{-2}$ with a ionization parameter of $\xi=0.39_{-0.17}^{+0.43} \mathrm{erg} \mathrm{cm} \mathrm{s}^{-1}$.

In Fig. 8 we show the confidence contours for the (log of the) ionization parameter and the column density of the ionized absorber from our best-fitting model IV. The absorber is only very slightly ionized, but the ionization parameter is well defined. Our best-fit model (IV) thus comprises a neutral zero-redshift plus ionized absorption at the redshift of 3C 109 for a total column density of $\sim 7.4 \times 10^{21} \mathrm{~cm}^{-2}$ which is in excellent agreement with the total obscuration inferred through infrared studies (and implying a column density of $\sim 7.7 \times 10^{21} \mathrm{~cm}^{-2}$ ).

By applying our best-fit absorption model (IV) to the broadband model C2 (see Table 1) we obtain a final fit to the broadband $0.4-10 \mathrm{keV}$ spectrum of $3 \mathrm{C} 109$ with $\chi^{2}=1272$ for $1358 \mathrm{dof}$. The same statistical quality is obtained by considering model $\mathrm{D}$ instead. No significant change is seen in the best-fit parameters reported in Table 1 and 2.

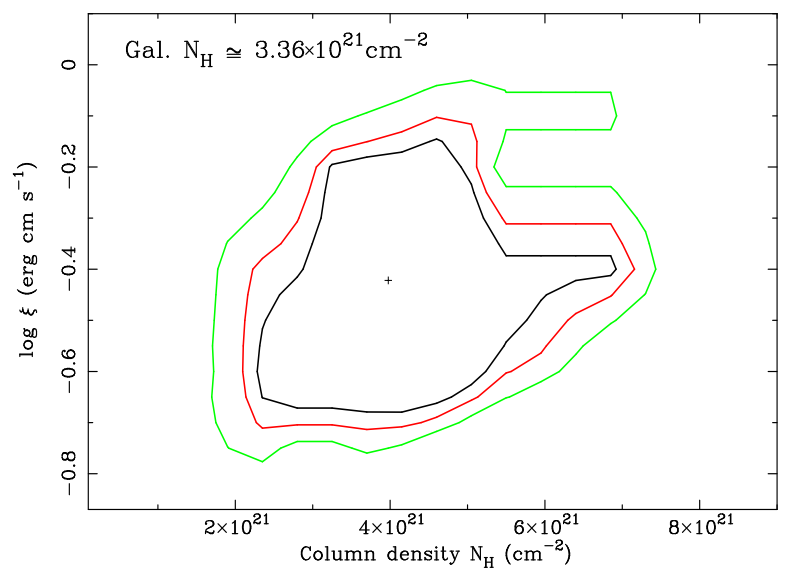

Figure 8. We show confidence contours for the ionization parameter and column density of the ionized absorber modelled through XSTAR (model IV, Table 2). The additional neutral and zero-redshift absorber provides an additional column density of $3.36_{-0.62}^{+0.41} \times 10^{21} \mathrm{~cm}^{-2}$ making the total column density of the order of $\sim 7.4 \times 10^{21} \mathrm{~cm}^{-2}$, in excellent agreement with studies in the optical and infrared.

\section{DISCUSSION}

We have presented results from a new $\sim 40 \mathrm{ks} X M M-N e w t o n$ observation of the BLRG/QSO 3C $109(\mathrm{z}=0.3056)$, one of the most $\mathrm{X}$-ray luminous sources within redshift 0.5 . The intrinsic $\mathrm{X}$-ray luminosity of the source is found to be $\sim 2-3 \times 10^{45} \mathrm{erg} \mathrm{s}^{-1}$ (depending on the adopted model) and compares well with previous $\mathrm{X}$-ray estimates, though the source is shown to be variable by a factor 2 at least on a few years timescale. The X-ray continuum is well represented by a relatively steep power law with $\Gamma \simeq 1.86$. Such a steep photon index is generally taken as an indication that a beamed component (often characterised by a flatter 1.5-1.7 photon index) contributes only marginally to the X-ray spectrum. However, this is not always the case, and an inner jet component cannot be ruled out on the basis of a steep X-ray slope only. The beamed component contribution to the $\mathrm{X}$-ray spectrum is particularly rel- 
evant because by combining the high $\mathrm{X}$-ray luminosity with the black hole mass estimate $\left(\sim 2 \times 10^{8} M_{\odot}\right)$, 3C 109 appears to be characterised by $L_{\mathrm{bol}} / L_{\mathrm{Edd}} \simeq 2.5$ which can be significantly reduced only by assuming that the $\mathrm{X}$-ray spectrum is strongly contaminated by a beamed inner jet component.

Fe emission is seen in the $6.3-7 \mathrm{keV}$ rest-frame band. A narrow core at $6.4 \mathrm{keV}$ is detected unambiguously, while additional curvature in the Fe $\mathrm{K}$ band can be modelled either in terms of reflection from the accretion disc or by invoking a partial covering scenario. If the disc reflection interpretation is correct, the disc surface is only slightly ionized and the emitting region must extend down to at least $\sim 26$ gravitational radii from the black hole. Such a solution allows one to place the inner disc radius as close as the innermost stable circular orbit around a Schwarzschild black hole (6 gravitational radii) but is also consistent with a truncated disc scenario in which the innermost 20-30 gravitational radii are missing, possibly replaced by thick and radiatively inefficient matter. The observer inclination is well constrained $\left(40^{\circ} \pm 8^{\circ}\right)$ and is consistent with 3C 109 being a BLRG/QSO borderline object, as indicated also by optical, infrared and radio studies. We measure a reflection fraction of the order of unity which is completely consistent with an overall $100 \mathrm{eV}$ equivalent width of the Fe line (narrow core plus disc line). The detection of substantial X-ray reflection from the accretion disc suggests that the $\mathrm{X}$-ray continuum is not significantly beamed away from the reflector and supports the estimated $L_{\text {bol }} / L_{\mathrm{Edd}} \simeq 2.5$ obtained by assuming isotropic X-ray emission. If instead partial covering is the right description of the spectral curvature in the Fe $\mathrm{K}$ band, about 20-30 per cent of the X-ray continuum source is covered by a large column $\left(\sim 5 \times 10^{23} \mathrm{~cm}^{-2}\right)$ of neutral gas $\left(\xi<0.5 \mathrm{erg} \mathrm{cm} \mathrm{s}^{-1}\right)$, and the intrinsic $X$-ray luminosity still requires $L_{\mathrm{bol}} / L_{\mathrm{Edd}}>1$ (but again in the hypothesis of negligible inner jet contribution).

In the soft band, the X-ray continuum is clearly absorbed by a column density in excess of the Galactic one, confirming previous findings both in the X-rays and at optical and infrared wavelengths (Allen \& Fabian 1992; Goodrich \& Cohen 1992; Allen et al. 1997; Rudy Puetter \& Mazuk 1999). We obtain a total column density of the order of $6-8 \times 10^{21} \mathrm{~cm}^{-2}$ which has to be considered as the sum of a zero-redshift neutral absorber and an in situ one at the redshift of $3 \mathrm{C} 109$. The best-fitting solution to the pn and MOS soft data strongly suggests that the in situ absorber is slightly ionized ( $\xi \sim 0.4 \mathrm{erg} \mathrm{cm} \mathrm{s}^{-1}$ ) with a non-zero ionization parameter preferred at more than the 99.9 confidence level. Our final result is that the zero-redshift neutral absorber contributes with a column density of $\sim 3.4 \times 10^{21} \mathrm{~cm}^{-2}$ to be added to a further $\sim 4.0 \times 10^{21} \mathrm{~cm}^{-2}$ from the ionized absorber at the redshift of 3C 109. The total column density compares remarkably well with the value of $\sim 7.7 \times 10^{21} \mathrm{~cm}^{-2}$ inferred from optical and infrared studies (Rudy, Puetter \& Mazuk 1999). Given the high luminosity of 3C 109, the low ionization of the absorber places it far from the central source (e.g. at the torus scale, suggesting that our line-ofsight is grazing the torus). This is also consistent with the observed large Balmer/Pashen decrement (Goodrich \& Cohen 1992; Rudy Puetter \& Mazuk 1999) in the broad-line region (BLR) making it likely that the in situ absorber is further away than the BLR with respect to the central engine. The obvious interpretation is that our line of sight is grazing the molecular torus, in good agreement with zeroth-order unification models (and with the inclination inferred from the broad Fe line).

The excellent agreement between the X-ray and the optical/infrared column density estimates implies that, if partial covering is indeed the right description of the hard spectrum, the addi- tional column of neutral gas $\left(\sim 5 \times 10^{23} \mathrm{~cm}^{-2}\right)$ cannot contribute to the optical/infrared absorption and must i) be dust-free (i.e. it lies within the dust sublimation radius), and ii) not obscure the broad lines (i.e. it lies within the broad-line-region). This places the partial coverer close to the $\mathrm{X}$-ray source and within the innermost $\simeq 0.1 \mathrm{pc}$. By combining this location with the source luminosity and the upper limit on the ionization parameter of the absorber we estimate a density of the order of $n>10^{11} \mathrm{~cm}^{-3}$ for the gas responsible for the partial covering. Given the relatively low covering fraction (20-30 per cent) and the large observed column density $\left(\sim 5 \times 10^{23} \mathrm{~cm}^{-2}\right)$ the absorbing matter is most likely distributed in compact clouds $<5 \times 10^{12} \mathrm{~cm}$ in size. Given the black hole mass estimate, the cloud size is of the order of $<0.17$ gravitational radii and to provide effective partial covering, the clouds must have a size comparable to that the main $\mathrm{X}$-ray emitting region. However, according to standard accretion models, $\mathrm{X}$-rays mostly come from the innermost 50 gravitational radii or so and thus, in order to provide a 20-30 per cent covering fraction, the absorber must comprise hundreds of clouds in the line of sight (therefore probably thousands of clouds if isotropically distributed), which seems quite extreme a requirement.

We conclude that, even if not unambiguous from a spectral point of view, the disc reflection (and broad Fe line) interpretation of the hard spectrum of 3C 109 is less contrived than the partial covering one and is also consistent with the high mass accretion rate implied by the inferred $L_{\mathrm{bol}} / L_{\mathrm{Edd}}$. If partial covering is ruled out, the contribution of the $\mathrm{X}$-ray reflection spectrum to the total flux implies a reflection fraction of the order of unity suggesting the a putative inner jet component does not contribute significantly in the $\mathrm{X}$-ray band and further supporting the idea that 3C 109 is radiating close to its Eddington luminosity due to accretion processes alone. The X-ray data do not rule out a truncated accretion disc in which the innermost 20-30 gravitational radii are missing or replaced by a radiatively inefficient flow, but this scenario is also at odds with the high estimated Eddington ratio. If the innermost regions of the accretion disc are instead present but highly ionized, little Fe line is produced there, reconciling the hard spectral shape with the huge $\mathrm{X}$-ray luminosity and the high mass accretion rate without the need to invoke a rather unusual partial covering scenario.

\section{ACKNOWLEDGEMENTS}

Based on observations obtained with XMM-Newton, an ESA science mission with instruments and contributions directly funded by ESA Member States and NASA. GM thanks Ross McLure for useful discussions on the black hole mass estimate in 3C 109 and Elena Belsole for discussions on the extend X-ray emission. GM thanks the PPARC for support. DRB is supported by the University of Arizona Theoretical Astrophysics Program Prize Postdoctoral Fellowship. This work was supported in part by the U.S. Department of Energy under contract number DE-AC02-76SF00515. ACF thanks the Royal Society for support. RRR thanks the College of the Holy Cross for support.

\section{REFERENCES}

Allen S.W., Fabian A.C., 1992, MNRAS, 258, 29

Allen S.W., Fabian A.C., Idesawa E., Inoue H., Kii T., Otani C., 1997, MNRAS, 286, 765

Antonucci R., 1993, ARA\&A, 31, 473 
Ballantyne D.R., Ross R.R., Fabian A.C., 2002, MNRAS, 332, L45

Ballantyne D.R., Fabian A.C., Iwasawa K., 2004, MNRAS, 354, 839

Ballantyne D.R., Fabian A.C., 2005, ApJ, 622, L97

Barthel P.D., 1989, ApJ, 336, 606

Beloborodov A.M., 1999, ApJ, 510, L123

Bennet C.L., Lawrence C.R., Burke B.F., Hewitt J.N., Mahoney J., 1986, ApJS, 61, 1

Bohlin R.C., Savage B.D., Drake J.F., 1978, ApJ, 224, 132

Burstein D., Heiles C., 1982, AJ, 87, 1165

Croston J.H., Hardcastle M.J., Harris D.E., Belsole E., Birkinshaw M., Worral D.M., 2005, ApJ, 626, 733

Done C., Mulchaey J.S., Mushotzky R.F., Arnaud K.A., 1992, ApJ, 395, 275

Elvis M., Willner S.P., Fabbiano G., Carleton N.P., 1984, ApJ, 280, 574

Elvis M. et al., 1994, ApJS, 95, 1

Elvis M., Risaliti G., Zamorani G., 2002, ApJ, 565, L75

Eracleous M., Halpern J.P., 1998, ApJ, 505, 577

Eracleous M., Sambruna R., Mushotzky R.F., 2000, ApJ, 537, 654

Fabian A.C., Rees M.J., Stella L., White N.E., 1989, MNRAS, 238, 729

Fabian, A. C., Iwasawa, K. 1999, MNRAS, 303, L34

Giovannini G., Feretti L., Venturi T., Lara L., Maracaide J., Rioja M., Spangler S.R., Wehrle A.E., 1994, ApJ, 435, 116

Gliozzi M., Sambruna R.M., Brandt W.N., Mushotzky R.F., Reacleous M., 2004, A\&A, 413, 139

Goodrich R.W., Cohen M.H., 1992, ApJ, 391, 623

Grandi S.A., Osterbrock D.E., 1978, ApJ, 220, 783

Grandi P., Malaguti G., Fiocchi M., 2006, ApJ in press (preprint astro-ph/0511784,

Haas M. et al., 2004, A\&A, 424, 531

Hardcastle M.J., Evans D.A., Croston J.H., 2006, submitted to MNRAS (preprint astro-ph/0603090)

Kallman T.R., Bautista M.A., ApJS, 133, 221

Laing R.A., Riley J.M., Longair M.S., 1983, MNRAS, 204, 151

Lawrence A., 1991, MNRAS, 252, 586

Lewis K.T., Eracleous M., Gliozzi M., Sambruna R.M., Mushotzky R.F., 2005, ApJ, 622, 816

Malizia A., Bassani L., Stephen J.B., di Cocco G., Fiore F., Dean A.J., 2003, ApJ, 589, L17

Meier D.L., 1999, ApJ, 522, 753

Meier D.L., 2001, ApJ, 548, L9

McLure R.J., Jarvis M.J., Targett T.A., Dunlop J.S., Best P.N., submitted to MNRAS (preprint astro-ph/0510121)

McLure R.J., Dunlop J.S., 2004, MNRAS, 352, 1390

Miniutti G., Fabian A.C., 2006, MNRAS, 366, 115

Nandra K., Pounds K., 1994, MNRAS, 268, 405

Narayan R., Yi I., 1995, ApJ, 444, 231

Orr M.J.L $\dot{i}$, Browne I.W.A., 1982, MNRAS, 200, 1067

Pointecouteau E., Arnaud M., Kaastra J., de Plaa J., 2004, A\&A, 423, 33

Porquet D., Reeves J.N., Uttley P., Turner T.J., 2004, MNRAS, 427, 101

Protassov R., van Dyk D.A., Connors A., Kashyap V.L., Siemiginowska A., 2002, ApJ, 571, 545

Ross R.R., Fabian A.C., 2005, MNRAS, 358, 211

Rudy R.J., Schmidt G.D., Stockman H.S., Tokunaga A.T., 1984, ApJ, 278, 530

Rudy R.J., Puetter R.C., Mazuk S., 1999, AJ, 118, 666
Sambruna R.M., Gliozzi M., Donato D., Maraschi L., Tavecchio F., Cheung C.C., Urry C.M., Wardle J.F.C., 2006, ApJ, 641, 717 Sanderson A.J.R., Finoguenov A., Mohr J.J., 2005, ApJ, 630, 191 Schlegel D.J., Finkbeiner D.P., Davis M., 1998, ApJ, 500, 525

Simpson C., 2003, New. Astron. Rev., 47, 211

Singal A.K., 1996, MNRAS, 278, 1069

Spinrad H., Marr J., Aguilar L., Djorgovski S., 1985, PASP, 97, 932

Sun M., Jones C., Murray S.S., Allen S.W., Fabian A.C., Edge A.C., 2003, ApJ, 587, 619

Willot C.J., Rawlings S., Blundell K.M., Lacy M., 2000, MNRAS, 316, 449

Woźniak O.R., Zdziarski A.A., Smith D., Madjeski G.M., Johnson W.M., 1998, MNRAS, 299, 449

Yaqoob T., Padmanabhan U., 2004, ApJ, 604, 63

Yee H.C.K., Oke J.B., 1978, ApJ, 226, 753 